\title{
Las prácticas externas extracurriculares: tutorización seguimiento como medida para incrementar su calidad.
}

\section{Extracurricular external practices: tutoring and supervision as a measure to increase quality.}

\author{
Ma Ángeles Valverde Arrebola \\ Jefa de Sección \\ Servicio de Cooperación Empresarial y Promoción de Empleo \\ Universidad de Málaga \\ mva@uma.es
}

Valderde Arrebola, Ma.A. (2016). Las prácticas externas extracurriculares:

tutorización y seguimiento como medida para incrementar su calidad. Revista Practicum, V1(1), 54-79 http://revistapracticum.com

Fecha de recepción: 25 de noviembre 2016

Fecha de revisión: 20 diciembre de 2016

Fecha de aceptación: 4 de enero de 2017 


\section{Resumen}

El programa de prácticas universitarias representa la oportunidad más clara para formar a los nuevos profesionales que requieren las organizaciones en este entorno tan complejo, no solo para el cometido del desarrollo de tareas sino también desde el punto de vista del desarrollo de las competencias profesionales (habilidades, actitudes y aptitudes). El presente estudio muestra el análisis de los resultados obtenidos en el primer Plan de Tutorización de Prácticas Extracurriculares que se implanta en la Universidad de Málaga, cuyo objeto es fomentar y cuidar las prácticas externas en las empresas para crear nuevos profesionales capaces de transformar las empresas en más competitivas e innovadoras. Siendo la población total sobre la que se ha implantado el proyecto de tutorización de 2001 estudiantes y 1236 empresas. Encontramos que el 82,61\% del total de titulaciones ofertadas en la Universidad de Málaga (Grado o Posgrado) han contado con prácticas extracurriculares, con una duración media aproximada de cada práctica de 4,5 meses.

\section{Abstract}

The university internship program represents the clearest opportunity to train the new professionals required by organizations in this complex environment, not only to promote the development of tasks but also from the point of view of the development of professional skills (skills, attitudes and aptitudes). The present study shows the analysis of the results obtained in the first Plan of Tutoring of Extracurricular Practices that is implanted in the Malaga University, whose object is to foment and to take care of the external practices in the companies to create new professionals capable of transforming the companies in more Competitive and innovative. The total population on which the tutoring project was implemented was 2001 students and 1236 companies. We found that $82.61 \%$ of the total degrees offered at the Málaga University (Degree or Postgraduate) have had extracurricular practices, with an approximate average duration of each practice is 4.5 months.

\section{Palabras claves}

Relación Universidad Empresa, Prácticas externas, Prácticas curriculares, Prácticas extracurriculares. 


\section{Key words}

revista

University Company Relationship, External Practices, Curricular Practices, Extracurricular Practices.

\section{Introducción}

El Espacio Europeo de Educación Superior estableció un nuevo escenario para las relaciones Universidad-Empresa poniendo un énfasis especial en las prácticas externas. Así, éstas han ido ganando en importancia en los últimos años ya que permiten a los alumnos obtener una experiencia profesional real y una formación práctica donde aplicar los conocimientos teóricos adquiridos, así como establecer relaciones que pueden ser de ayuda en su incorporación al mercado laboral, dándose a conocer a las empresas del sector.

Asimismo, las prácticas suponen para las empresas una interesante oportunidad de formar a estudiantes que pueden constituir una cantera de personal especializado para su contratación posterior.

Con la publicación del Real Decreto 1707/2011, de 18 de noviembre derogado por el Real Decreto 592/2014, de 11 de julio, por el que se regulan las prácticas académicas externas de los estudiantes universitarios se establecen dos modalidades de prácticas: curriculares y extracurriculares. La principal diferencia entre ellas radica en que formen parte del Plan de Estudios como una asignatura más (curriculares) o no.

Desde el Servicio de Cooperación Empresarial y Promoción de Empleo de la Universidad de Málaga, y al amparo de la nueva normativa, se diseña el procedimiento de realización de las prácticas externas realizando un trabajo conjunto con los Centros. Así se pone de manifiesto la necesidad de homogeneizar los criterios de control y seguimiento de la modalidad extracurricular a fin de ofrecer una mayor calidad que permita, en su caso, el reconocimiento de las prácticas extracurriculares como "convalidables" por curriculares, facilitando así a los Centros la captación de empresas. 


\section{Contexto normativo}

En nuestro ordenamiento, la primera regulación de las prácticas para estudiantes universitarios se abordó en el Real Decreto 1497/1981, de 19 de junio, sobre Programas de Cooperación Educativa. En líneas generales, el objetivo fundamental de esta norma era conseguir una formación integral del alumno universitario a través de programas de cooperación educativa con las empresas para la formación de los alumnos de los dos últimos cursos de una Facultad, Escuela Técnica Superior o Escuela Universitaria concreta o para un grupo de estos centros con características comunes. El programa no establecía relación contractual alguna sobre el estudiante y la empresa, toda vez que, por su naturaleza, dicha relación era estrictamente académica y no laboral. En esta normativa ya se establecía la figura del "tutor" en la empresa, si bien no se hacía ninguna referencia a cómo debía articularse dicha tutorización.

El Estatuto del Estudiante Universitario, aprobado por Real Decreto $1791 / 2010$, de 30 de diciembre, reconoce en su artículo 8 el derecho de los estudiantes de Grado a «disponer de la posibilidad de realización de prácticas, curriculares o extracurriculares, que podrán realizarse en entidades externas $y$ en los centros, estructuras o servicios de la Universidad, según la modalidad prevista y garantizando que sirvan a la finalidad formativa de las mismas» (apartado f) y a «contar con tutela efectiva, académica y profesional (...) en las prácticas externas que se prevean en el plan de estudios» (apartado g). Con mayor detalle, el artículo 24 de este Estatuto regula las prácticas académicas externas, sus clases y sus características generales, así como la extensión de su realización a todos los estudiantes matriculados en cualquier enseñanza impartida por las universidades o centros adscritos a las mismas.

Así, dada la importancia que la nueva ordenación de los estudios universitarios conceden a la realización de "prácticas externas", se aprueba el Real Decreto 592/2014 de 11 de julio, por el que se regulan las prácticas académicas externas de los estudiantes universitarios, que establece un marco más acorde con lo establecido en la legislación vigente y que desarrolla aspectos tales como los objetivos de las prácticas, las entidades colaboradoras y los destinatarios, requisitos, tutorías y contenidos de los convenios de cooperación educativa. 
evista $\frac{\mathrm{O}}{\mathrm{f}} \mathrm{b}$ practicum

En esta línea normativa, la Universidad de Málaga de conformidad con lo dispuesto en su Normativa de Prácticas Externas, aprobada por Consejo de Gobierno de 13 de marzo de 2013, diseña un procedimiento para realizar la tutorización y seguimiento de las prácticas extracurriculares, cuya organización corresponde al Servicio de Cooperación Empresarial y Promoción de Empleo.

El siguiente gráfico muestra la evolución que se aprecia en el número de estudiantes que han realizado prácticas en los últimos cuatro cursos académicos. De su observación podemos afirmar que existe una tendencia estable al alza curso a curso, tras un leve receso en el curso 2014-2105.

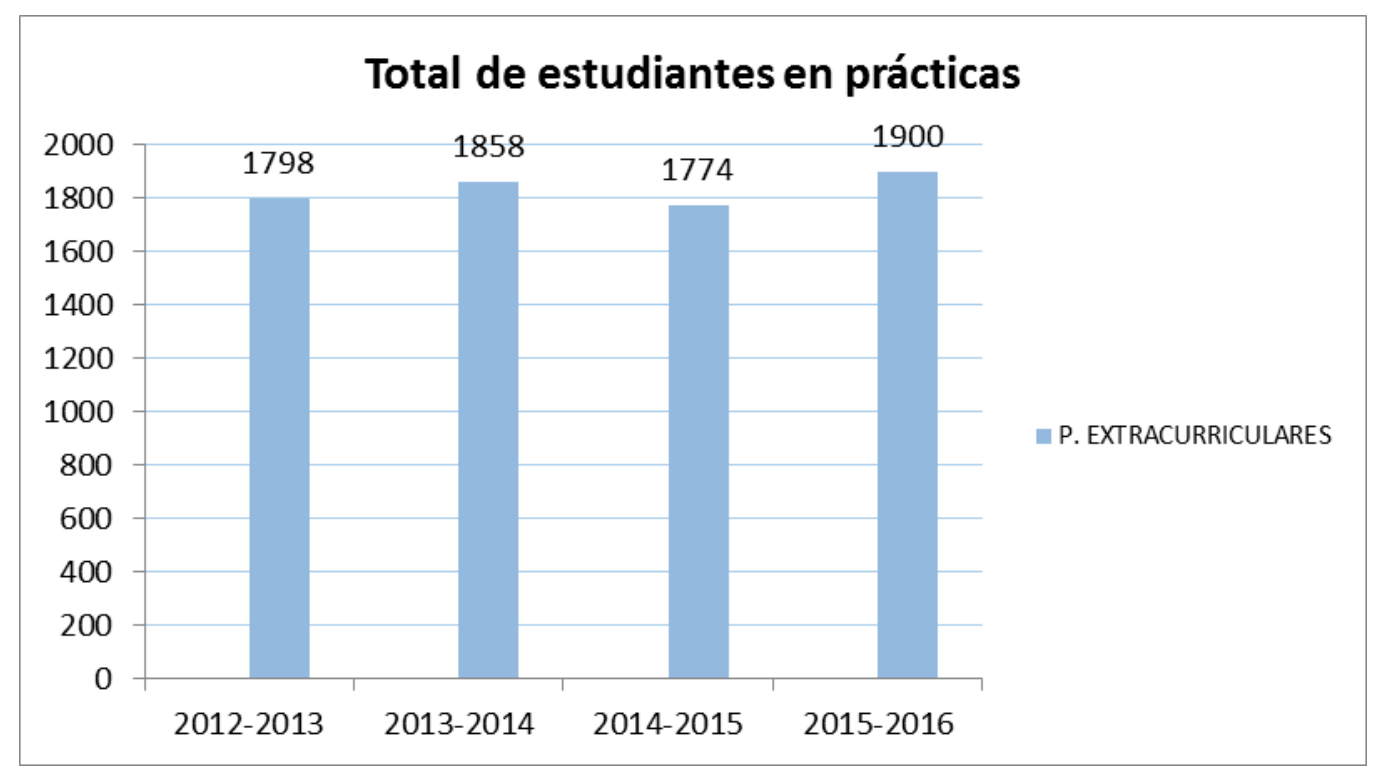

Tabla 1. Número de estudiantes que realizan las prácticas extracurriculares.

Elaboración propia.

Debemos señalar que los datos arriba aportados se miden desde 1 de octubre a 30 de septiembre, mientras que el rango temporal objeto del presente estudio abarca desde 1 de septiembre de 2015 a 19 de septiembre de 2016, ascendiendo a 2001 prácticas que han sido objeto de análisis.

\section{Objetivos}

Dada la importancia que la nueva ordenación de los estudios universitarios concede a la realización de "prácticas externas", el Real Decreto 592/2014 de 11 de julio, por el que se regulan las prácticas académicas externas de los estudiantes universitarios, establece un 
marco muy detallado que desarrolla aspectos tales como los objetivos de las prácticas, las entidades colaboradoras y los destinatarios, requisitos, tutorías y contenidos de los convenios de cooperación educativa.

Igualmente, debemos señalar que los recientes procesos de evaluación de las titulaciones universitarias, realizados por la Dirección de Evaluación y Acreditación (DEVA), dependiente de la Agencia Andaluza del Conocimiento, mostraban un evidente interés, no sólo en los resultados de las prácticas curriculares, sino que también se solicitaban resultados sobre prácticas extracurriculares.

En esta línea, la Universidad de Málaga, a través del Servicio de Cooperación Empresarial y Promoción de Empleo, puso en marcha en el curso 2015-2016 un "programa de tutorización" de las prácticas extracurriculares.

El objetivo es la implementación de un sistema de tutorización y recogida de información que permita mejorar la calidad de las prácticas que ofrecemos a nuestros estudiantes, así como obtener "feed back" de las empresas sobre la adecuación de la formación de los estudiantes a sus necesidades.

Con la puesta en marcha de este proyecto se pretende:

- Incrementar la calidad de las prácticas extracurriculares.

- Familiarizar a los agentes que intervienen en el proceso con la cultura de la evaluación y de la Calidad.

- Detectar desviaciones en los objetivos de las prácticas externas.

- Aumentar la vinculación y la participación de las empresas e instituciones participantes en los programas, haciéndoles sentir partícipes de la formación.

\section{Metodología para la tutoría de alumnos en prácticas externas extracurriculares}

El proyecto fue elaborado por el personal adscrito al Servicio de Cooperación Empresarial y Promoción de Empleo (Unidad de Prácticas) en el marco del "Proyecto para desarrollo de una estrategia 
de Empleabilidad en la Universidad de Málaga", publicado por Resolución Rectoral de 10 de abril de 2015.

\subsection{Medios técnicos y personales}

El procedimiento fue planteado mediante la explotación de las posibilidades que nos ofrece la herramienta informática ICARO, desarrollada por la Fundación Mediterránea, y que constituye un portal de gestión integral de prácticas y empleo, utilizado actualmente por las Universidades Públicas Andaluzas, la Universidad Politécnica de Cartagena y la Universidad Autónoma de Madrid.

Para dar respuesta al diseño del proyecto, ICARO fue complementado por medios ofimáticos comunes. Para ello se solicitaron a los administradores de la plataforma las mejoras y modificaciones necesarias. En ningún momento se plantea la utilización de nuevas herramientas por razones presupuestarias y de tiempos de ejecución.

En cuanto a los medios personales utilizados se dedicaron cinco personas al diseño del proyecto con perfiles profesionales diferentes (responsables de la Unidad de Prácticas, personal técnico informático, y personal con experiencia en el seguimiento de programas de prácticas).

La ejecución del programa fue asignada a dos personas cuyo perfil laboral corresponde a "Técnicos en Orientación Profesional", con experiencia en tutorización de programas de prácticas.

\begin{tabular}{|c|c|}
\hline \multicolumn{2}{|l|}{ PERSONAL ASIGNADO } \\
\hline Diseño del proyecto & 5 \\
\hline Recogida de datos & 2 \\
\hline Tratamiento de datos y elaboración de informe de resultados & 1 \\
\hline \multicolumn{2}{|l|}{ TEMPORIZACIÓN } \\
\hline Diseño del proyecto y adaptaciones informáticas & $5 / 2015$ \\
\hline Pruebas piloto & $17 / 6$ a $31 / 82015$ \\
\hline Recogida de datos & $1 / 9 / 215$ a $19 / 9 / 2016$ \\
\hline \multicolumn{2}{|l|}{ METODOLOGÍA } \\
\hline Tipología de encuestas & Descriptiva, respuesta cerrada \\
\hline Herramienta de recogida de datos & Icaro \\
\hline
\end{tabular}


Tabla 2. Resumen de recursos. Elaboración propia.

\subsection{Procedimiento}

Se toma como referencia para iniciar el proyecto de tutorización el día 17 de junio, desarrollándose en dos fases:

Primera fase: estudiantes que iniciaron sus prácticas a partir del día 17 de junio. Se inició una tutorización con carácter piloto de forma no obligatoria con la finalidad de detectar fallos en el procedimiento. Las pruebas se realizaron con un total de 278 estudiantes.

Segunda fase: estudiantes que iniciaron sus prácticas a partir del 1 de septiembre. El procedimiento se implantó de forma obligatoria a partir de esta fecha.

Con esta referencia, la población total sobre la que se ha implantado el proyecto de tutorización fue de 2001 estudiantes y 1236 empresas.

Los datos, tanto en estudiantes como en empresas, hace referencia a "prácticas iniciadas" durante el intervalo objeto de estudio, teniendo en cuenta que la duración media aproximada de cada práctica es de 4,5 meses (Fuente: Icaro: 3/10/16).

La figura 1 muestra la distribución por sexo de las prácticas objeto del estudio, siendo mujeres en $57 \%$ del total de estudiantes que han realizado prácticas y hombres el $42,43 \%$.

PRÁCTICAS REALIZADAS: DISTRIBUCIÓN POR SEXO

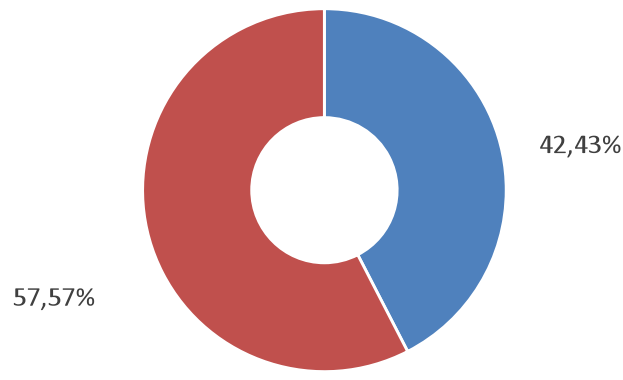

- Hombres - Mujeres

Figura 1. Datos de las prácticas realizadas según el sexo. Elaboración propia. 
La figura 2 muestra la distribución del número de prácticas teniendo en cuenta el tipo de titulación. El 89,26\% de las prácticas iniciadas corresponden a los nuevos títulos de Grado o Posgrado, quedando aún un 10,74\% de estudiantes procedentes de los antiguos títulos de Licenciatura o Diplomatura.

\section{ALUMNOS POR TIPO DE TITULACIÓN}

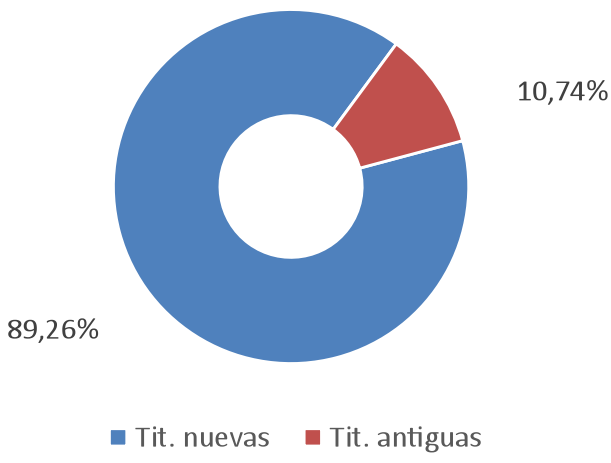

Figura 2. Datos de población: Tipo de titulación. Elaboración propia.

La Figura 3 muestra la distribución mensual de las 2001 prácticas iniciadas durante el periodo estudiado. Los datos muestran un receso evidente durante los meses de diciembre a febrero que constituyen períodos de exámenes. El mayor incremento se produce durante los meses de verano. El descenso durante el mes de agosto se explica por el cierre de los servicios universitarios por vacaciones, por lo que aquellas prácticas cuya incorporación no se haya gestionado a fecha 30 de julio queda aplazado su inicio hasta septiembre. El volumen medio mensual de prácticas está en torno a 150. 
Las prácticas externas extracurriculares: tutorización y seguimiento como medida para incrementar su calidad.

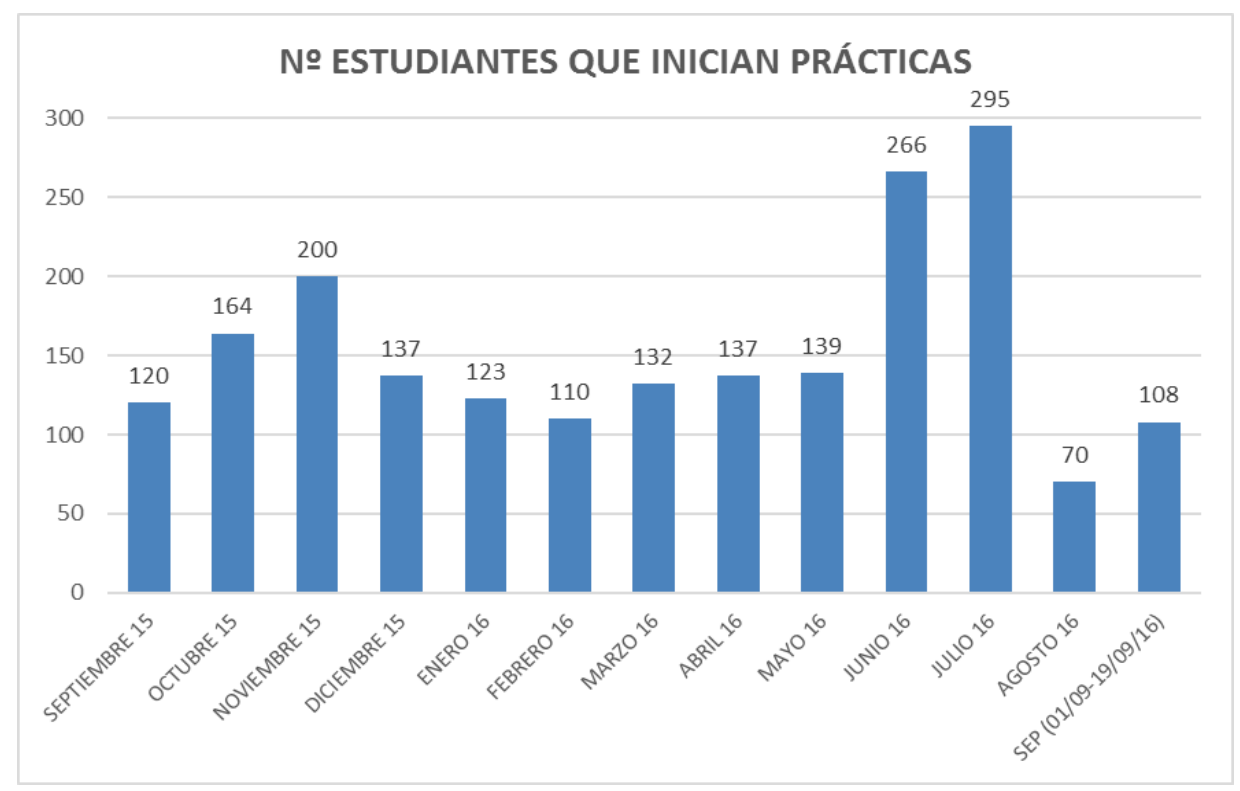

Figura 3. Distribución de estudiantes participantes en proyecto. Elaboración propia.

Un total de 1236 empresas que ofertaron las 2001 prácticas estudiadas, lo que indica que las empresas ofertan más de 1 práctica bien simultánea o sucesivamente.

En la figura 4 se evidencia la distribución mensual por empresa que inician las prácticas que han ofertado en el período objeto de estudio. La distribución coincide con la mostrada para los alumnos, siendo los meses de verano los que registran un evidente incremento.

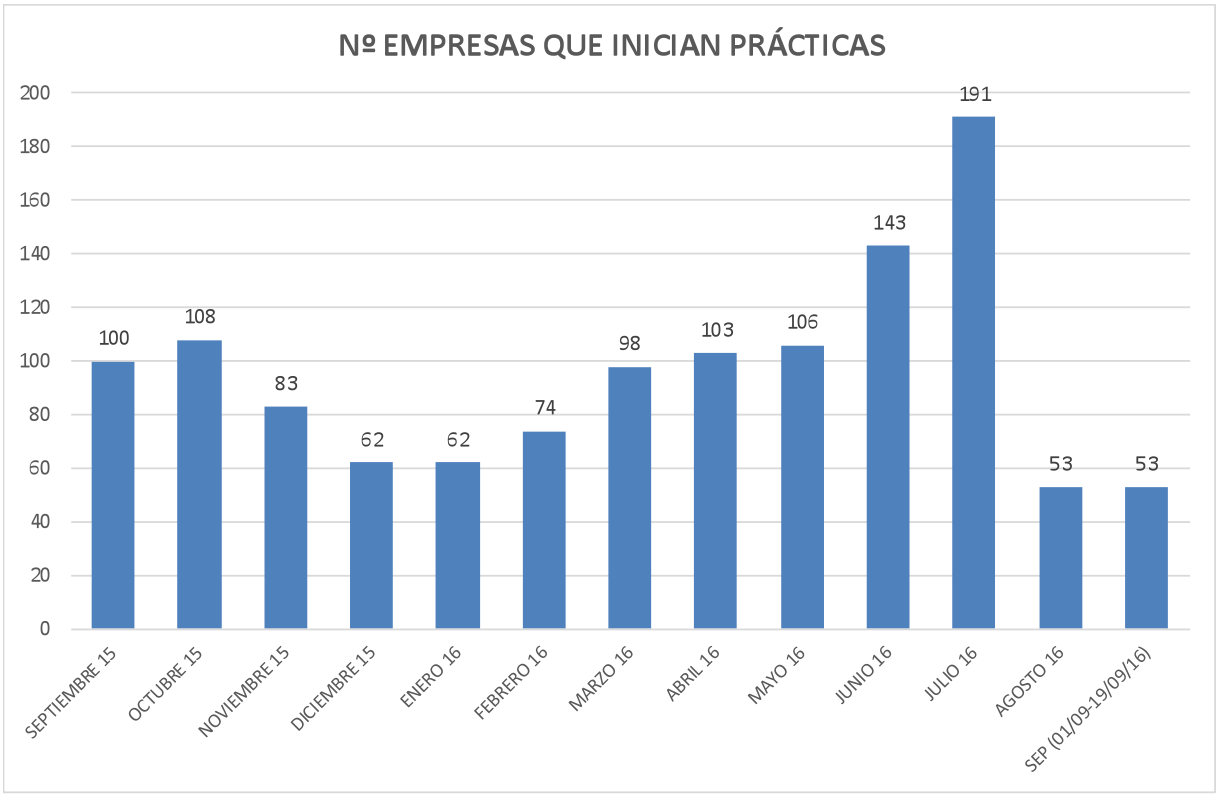

Figura 4. Distribución mensual de empresas participantes en el proyecto. Elaboración propia 


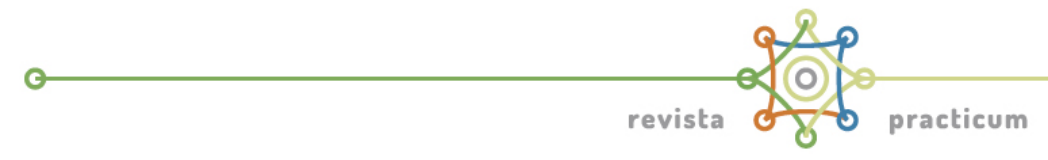

Para analizar las titulaciones más demandadas por las empresas e instituciones debemos tener en cuenta que las ofertas de prácticas extracurriculares no se realizan de forma obligatoria para una sola titulación, sino que es frecuente que cada oferta incluya 2 o más titulaciones con contenidos afines al perfil que se oferta.

Así, podemos afirmar que el $82,61 \%$ del total de titulaciones ofertadas en la Universidad de Málaga (Grado o Posgrado) han contado con prácticas extracurriculares.

Igualmente, considerando el nivel de los estudios que finalmente han disfrutado de prácticas extracurriculares el 86'96\% ha correspondido a titulaciones consideradas de primer nivel (Grado, Licenciatura, Diplomatura, Ingeniería e Ingeniería Técnica), el $12,14 \%$ corresponden al nivel Master y $0,90 \%$ a Doctorado.

TITULACIONES CON PRÁCTICAS

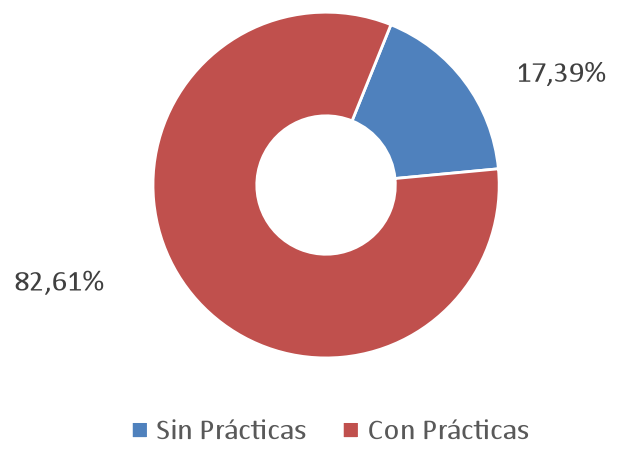

Figura 5. Titulaciones con prácticas realizadas. Elaboración propia

En cuanto a la distribución por titulaciones hay que señalar la gran dispersión existente en el reparto de las prácticas realizadas.

Para facilitar la comprensión se han realizado 3 cuadros diferentes, en el primero se muestran las titulaciones que han tenido al menos 20 prácticas extracurriculares, en el segundo entre 5 y 19 y finalmente entre 1 y 4 prácticas.

Debemos destacar que el $50 \%$ (1002) de las prácticas realizadas se concentran en 10 titulaciones, todas de la rama de conocimiento Ciencias Sociales y Jurídicas, siendo la que más 
Las prácticas externas extracurriculares: tutorización y seguimiento como medida para incrementar su calidad.

prácticas concentra el Grado en Administración y Dirección de Empresas (282) que, incluso casi duplica la siguiente con mayor número de prácticas, Grado en Economía (144).

La titulación con más prácticas realizadas de la rama Ingenierías y Arquitectura fue el Grado e Ingeniería Mecánica con 36 prácticas, seguida por el Grado en Ingeniería de Telecomunicación con 28.

En la rama Ciencias la titulación mejor posicionada fue el Grado en Ciencias Ambientales con 22 prácticas realizadas, descendiendo a 16 para la siguiente titulación del área, Grado en Biología.

En la rama Arte y Humanidades la titulación con mayor número de prácticas fue el Grado en Estudios Ingleses (16), seguida muy de cerca por el Grado en Traducción e Interpretación (12).

Finalmente, la rama Ciencias de la Salud la titulación mejor posicionada fue el Grado en Podología (3).

Los datos se evidencian de forma clara en los cuadros siguientes.

DISTRIBUCIÓN POR TITULACIONES (MAYOR O IGUAL QUE 20)

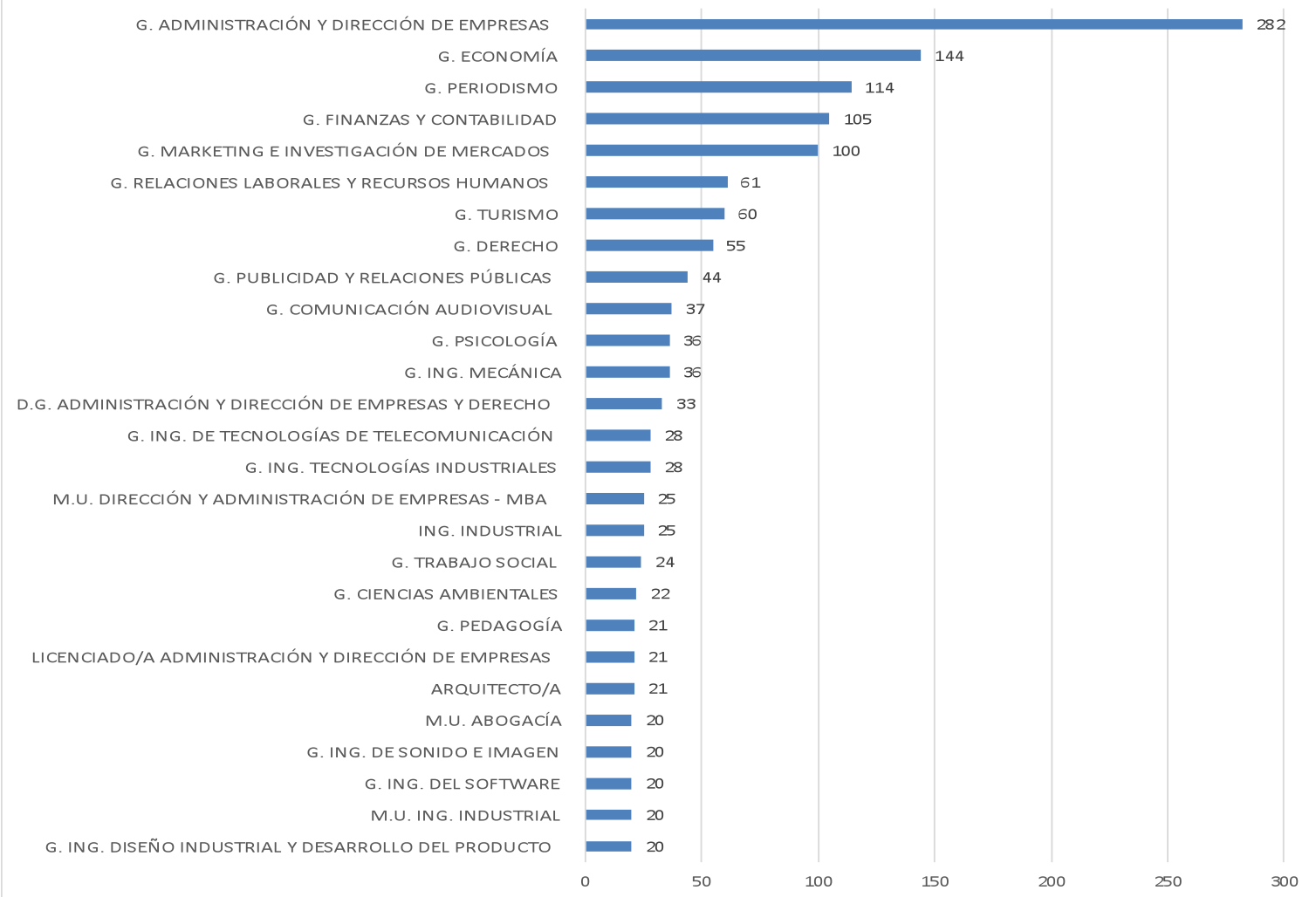

Figura 6. Distribución de prácticas realizadas. Elaboración propia. 

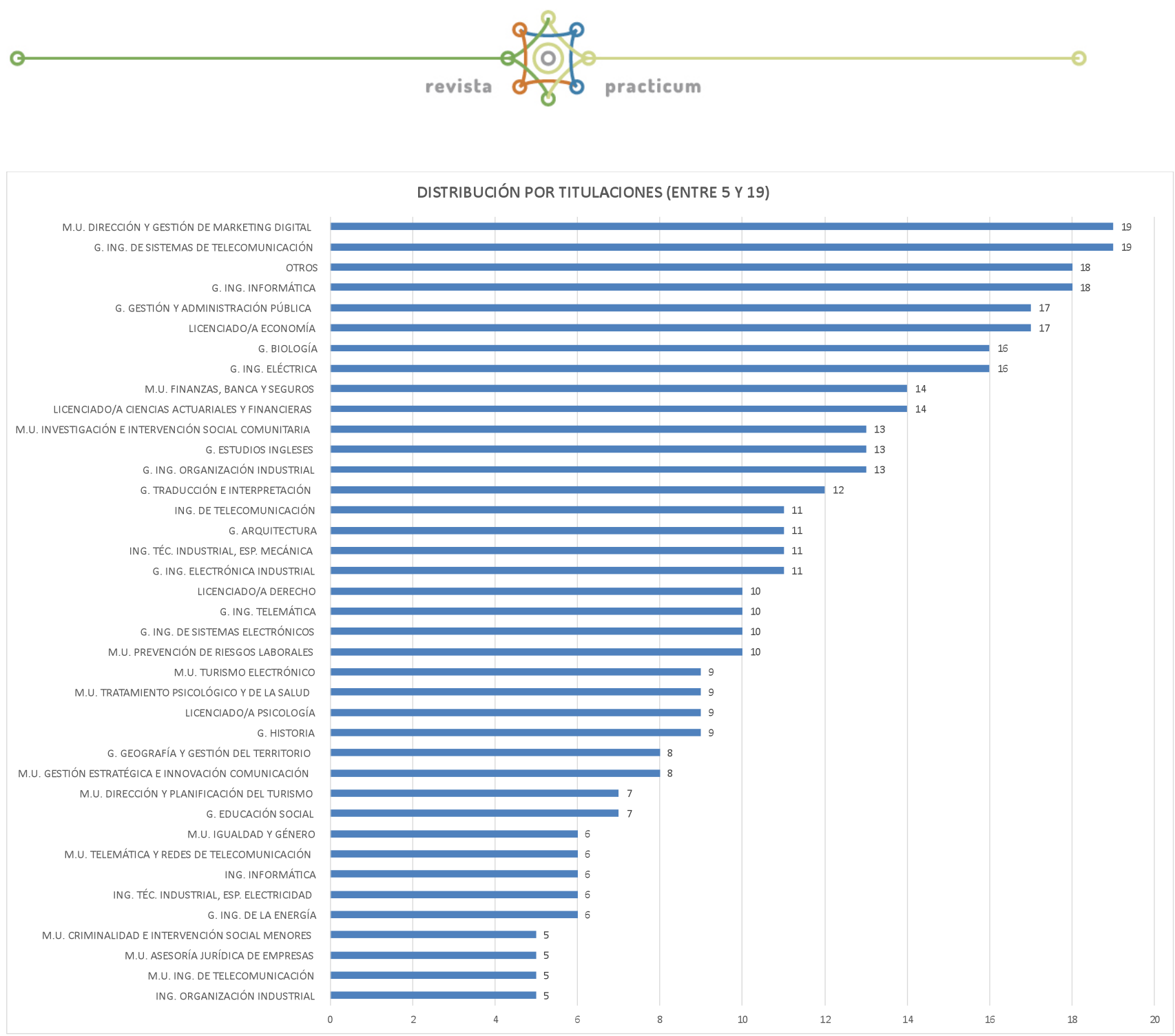

Figura 7. Distribución de prácticas realizadas II. Elaboración propia. 


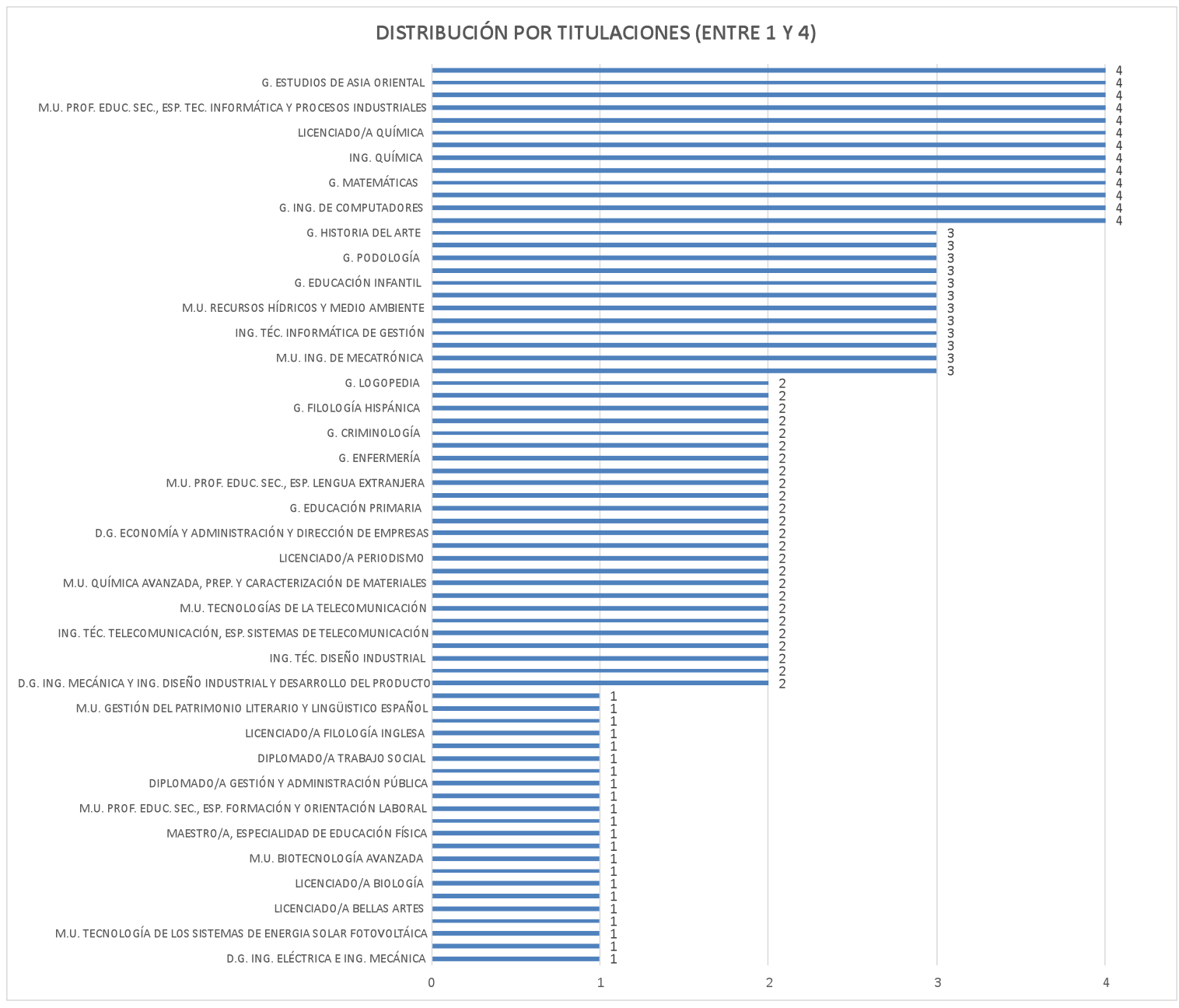

Figura 8. Distribución de prácticas realizadas III. Elaboración propia

\subsection{Descripción del proceso}

En los apartados anteriores se analiza la población potencial sobre la que se realiza el estudio: 2001 estudiantes y 1236 empresas.

El estudio se realiza finalmente con la respuesta de 1260 estudiantes y 1003 empresas que realizaron las encuestas de valoración.

Considerando que las prácticas deben tener una duración de entre 2 y 6 meses, la media, según los datos procedentes de la plataforma ICARO, fue de 4,5 meses por práctica. Así una práctica iniciada en el mes de julio de 2016 es posible que aún no tenga disponibles sus informes, puesto que su realización dependerá de la duración de la misma. Y de la misma forma los informes completados en los meses septiembre a diciembre de 2015 proceden de prácticas iniciadas en 
los meses de junio o julio, cuando el proceso estaba en fase piloto. Por ello, no podemos establecer una tasa de respuesta real y directa.

Otra cuestión importante que afecta al nivel de respuesta se explica porque, si bien las tareas que conlleva el programa de tutorización de las prácticas se configuran como un elemento obligatorio para el estudiante, el hecho de que las prácticas extracurriculares sean de realización voluntaria y no conlleven una calificación sino una certificación, hace muy difícil obligar al estudiante a que complete el proceso, siendo sólo aquellos que van a solicitar el reconocimiento de créditos los que tienen tendencia a realizar las encuestas y responder a las tutorías.

En el caso de las empresas, es aún más complicado, ya que la única forma de obtener los informes es convencerlos de que deben hacerlo.

A pesar de todo ello, en el Servicio de Cooperación Empresarial y Promoción de Empleo estamos satisfechos con el nivel de respuesta obtenido, que ha ido evolucionando favorablemente a medida que tanto empresas como estudiantes conocían el programa y las tutoras trabajaban con ellos.

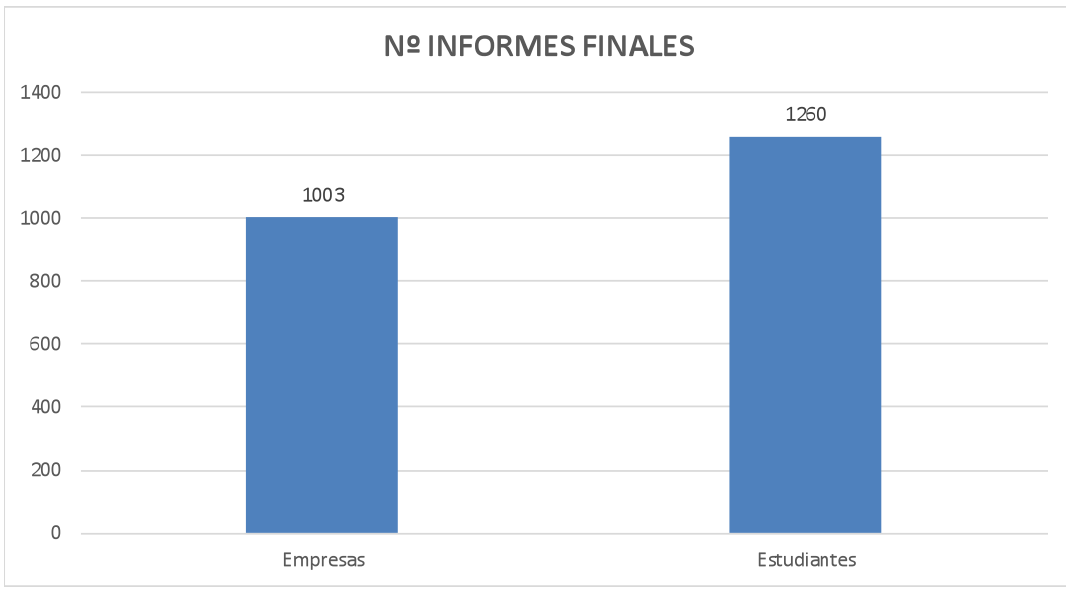

Figura 9. Informes completados. Elaboración propia.

\subsection{El procedimiento de tutorización: Desarrollo}

\section{A) PRIMER CONTACTO}

Durante el primer mes de prácticas se estableció contacto tanto con los estudiantes, como con los tutores en las empresas. Se 
realizó mediante correo electrónico informándoles de los pormenores del procedimiento de tutorización y comunicándoles los datos de contacto de la persona responsable de la tutorización en la Universidad.

De esta forma, se pretendió dar confianza a ambas partes y presentar un enlace entre la empresa y la Universidad. Se trataba de superar la percepción de que las prácticas extracurriculares no son supervisadas por nadie.

\section{B) SEGUIMIENTO INTERMEDIO}

A la mitad, aproximadamente, del periodo de cada práctica se estableció una tutoría con carácter voluntario, es decir, no se tuvo en cuenta su realización en el momento de emitir la acreditación de las prácticas. A través de esta tutoría el estudiante respondió a 5 a cuestiones relacionadas con la práctica (funciones, expectativas, cumplimiento del proyecto formativo...). Este cuestionario se realizó en formato "encuesta", de respuesta abierta utilizando el módulo al efecto en ICARO.

1.- ¿Se cumplen las condiciones acordadas en la oferta de prácticas?

2.- ¿Te has adaptado al entorno laboral donde realizas tus prácticas?

3.- ¿La atención que recibes de tu tutor en la empresa es adecuada?

4.- ¿Estas satisfecho con el desarrollo de tus prácticas hasta el momento?

5.- ¿Deseas reseñar alguna observación?

Figura 10. Modelo de encuesta intermedia. Elaboración propia.

En el cuadro siguiente podemos observar la evolución mensual de la realización de estos cuestionarios. Finalmente, se completaron a un total de 1095 seguimientos intermedios, frente a un total de 1260 cuestionarios finales, lo que supone un $\mathbf{8 6 , 9 \%}$. Si bien este contacto se planteó como voluntario, la labor de las tutoras insistiendo vía telefónica o correo electrónico hizo que se obtuviera una alta tasa de respuesta, reforzando la percepción de supervisión.

Podemos observar cómo el nivel de respuesta es constante, excepto los meses de realización de exámenes (enero y febrero) en que desciende claramente. Igualmente, debemos señalar una relación directa entre los meses de más alta respuesta (diciembre, julio y septiembre 16) y la dedicación de las tutoras. Durante estos períodos se hizo un especial esfuerzo de seguimiento. 


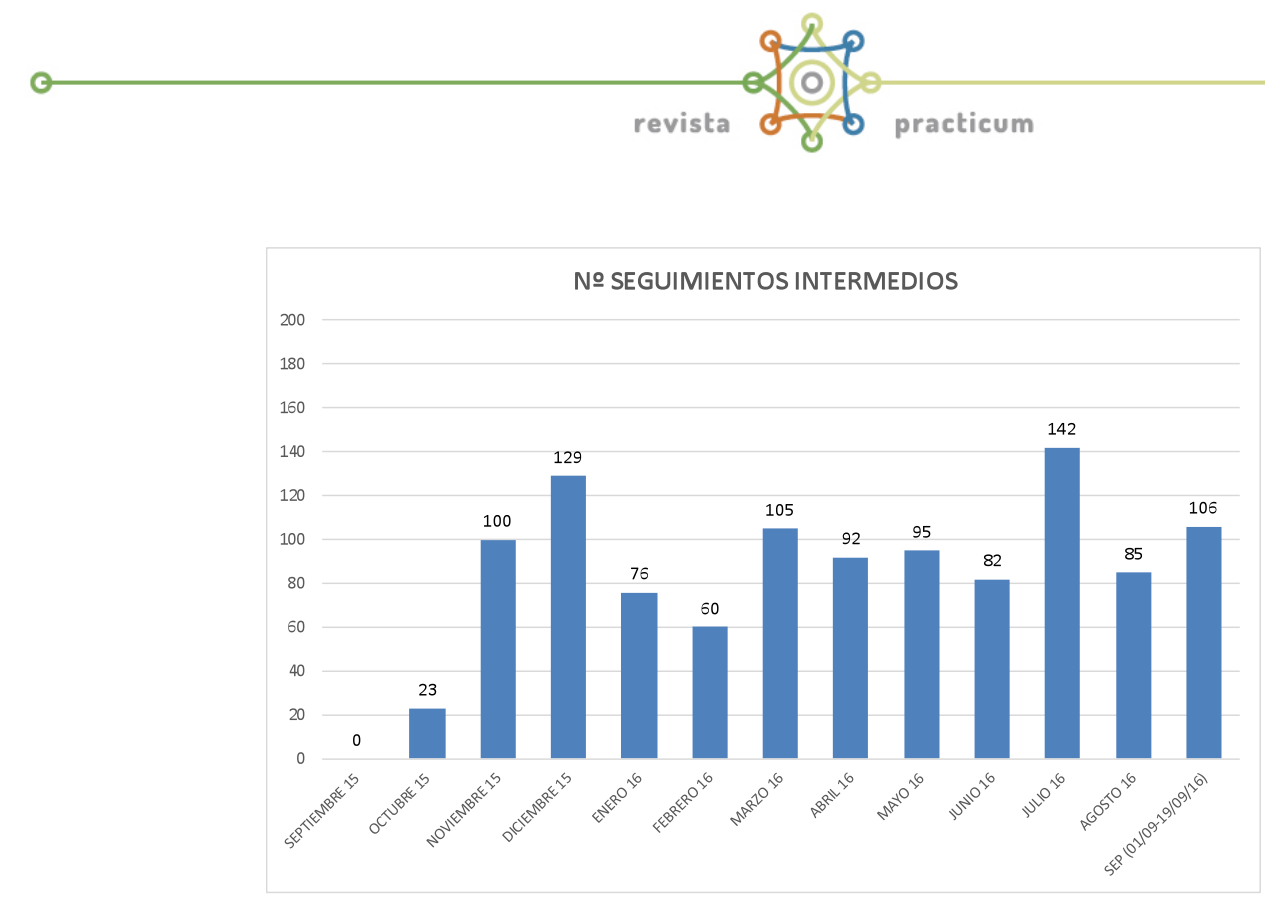

Figura 11. Evolución mensual de seguimientos intermedios. Elaboración propia.

Las respuestas permitieron detectar anomalías en el desarrollo de las prácticas para tratar de solucionarlas, evitando así la renuncia del estudiante o la rescisión anticipada por parte de la empresa.

Al detectar alguna desviación en alguna de las cuestiones, las tutoras contactaban con el estudiante, que muy frecuentemente se mostraba sorprendido, reconociendo en numerosos casos que no pensaban que nadie en la Universidad leyera los cuestionarios.

Tras la sorpresa inicial, la acogida a la iniciativa fue buena y se sinceraban con las tutoras con relativa facilidad. Para lograrlo fue necesario que las tutoras les transmitieran confianza y les convencieran de la absoluta confidencialidad de sus conversaciones.

Las tutorías intermedias se iniciaron de forma telefónica derivando en tutorías presenciales si así lo requería el estudiante o la tutora.

Durante toda la duración de sus prácticas el estudiante contó con un número flexible de acciones directas de intervención dependiendo de sus propias necesidades.

$\mathrm{Si}$ bien al principio el requerimiento de atenciones personalizadas fue anecdótico, a partir del mes de mayo se 
dispara a 105 alcanzando en julio las 164. En agosto caen por el cierre de los servicios universitarios y a fecha 19 de septiembre se habían realizado 75 atenciones directas.

El objeto es aclarar dudas, solucionar problemas con las empresas, o con los propios estudiantes, intermediar en posibles conflictos o incumplimientos de proyectos formativos, ayudar en la adaptación del estudiante a la empresa... La vía de solución de problemas fue siempre el diálogo con las partes: estudiantes y tutor en la empresa.

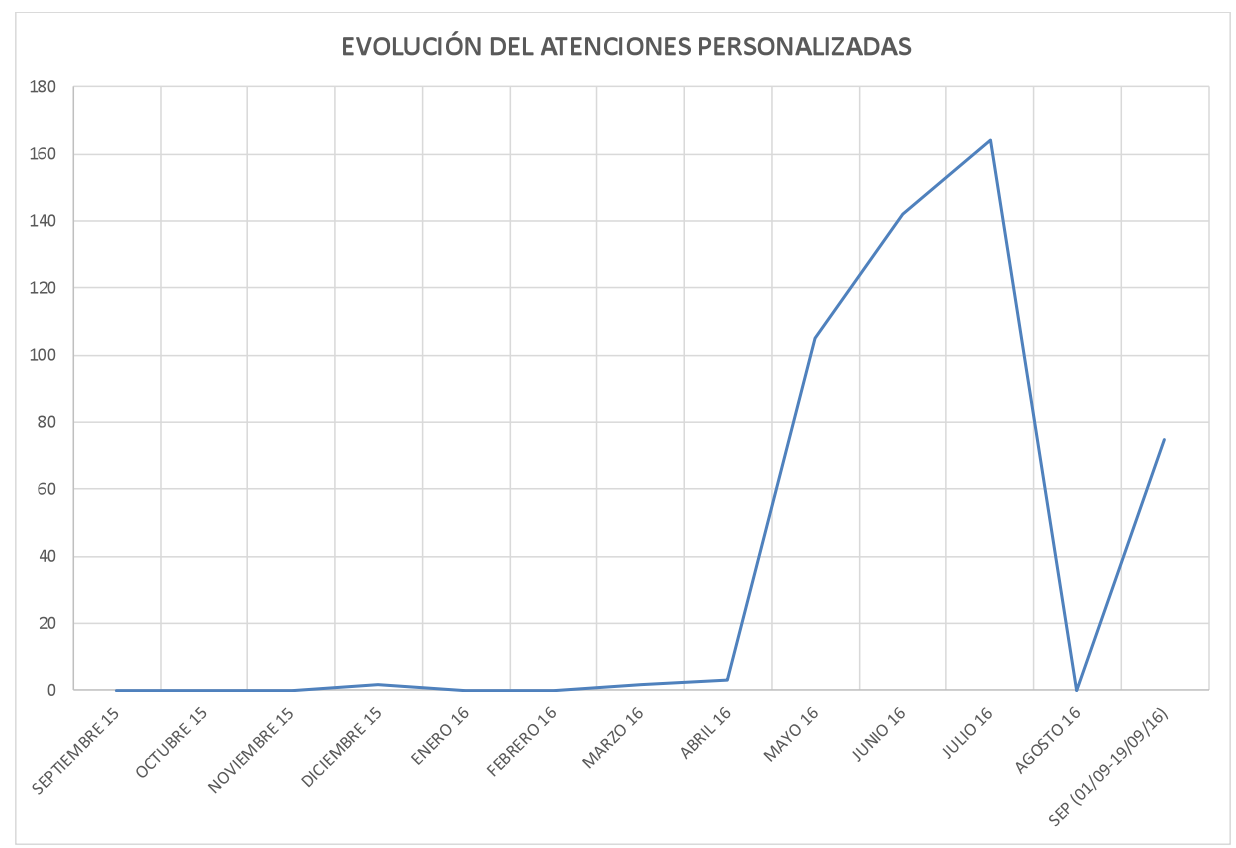

Figura 12. Evolución mensual de atenciones personalizadas. Elaboración propia.

No se debe interpretar este espectacular incremento como una mayor afluencia de problemas, ya que las consultas realizadas fueron, en su mayoría, sobre cuestiones administrativas. Podemos decir que el aumento de la demanda de tutorías presenciales es consecuencia de la difusión del programa, bien entre los propios estudiantes o como consecuencia de acciones informativas en redes sociales, charlas de orientación e incluso publicación en prensa.

\section{C) ENCUESTA FINAL}

Siete días antes de la finalización de la práctica tanto el estudiante como su tutor en la empresa tuvieron disponible el informe final de la misma cuya realización tiene carácter obligatorio. Este informe se completó a través de la plataforma ICARO. El Informe contiene información sobre el desarrollo de sus prácticas y una breve encuesta 
revista $\frac{0}{8}$ practicum

que nos permitirá conocer la satisfacción de ambas partes con las prácticas.

La acreditación del estudiante sólo se emitió en el caso de que se hubieran completado todas las acciones obligatorias.

El siguiente cuadro muestra una tendencia paralela en el número de informes finales completados en estudiantes (1260) y empresas (1003). Los meses que muestran mayor número de informes presentados corresponden a diciembre, mayo, junio y julio. Estos meses corresponden a fechas de finalización de estudios y de mayor afluencia en la solicitud de certificaciones de créditos.

EVOLUCIÓN DEL NÚMERO DE INFORMES

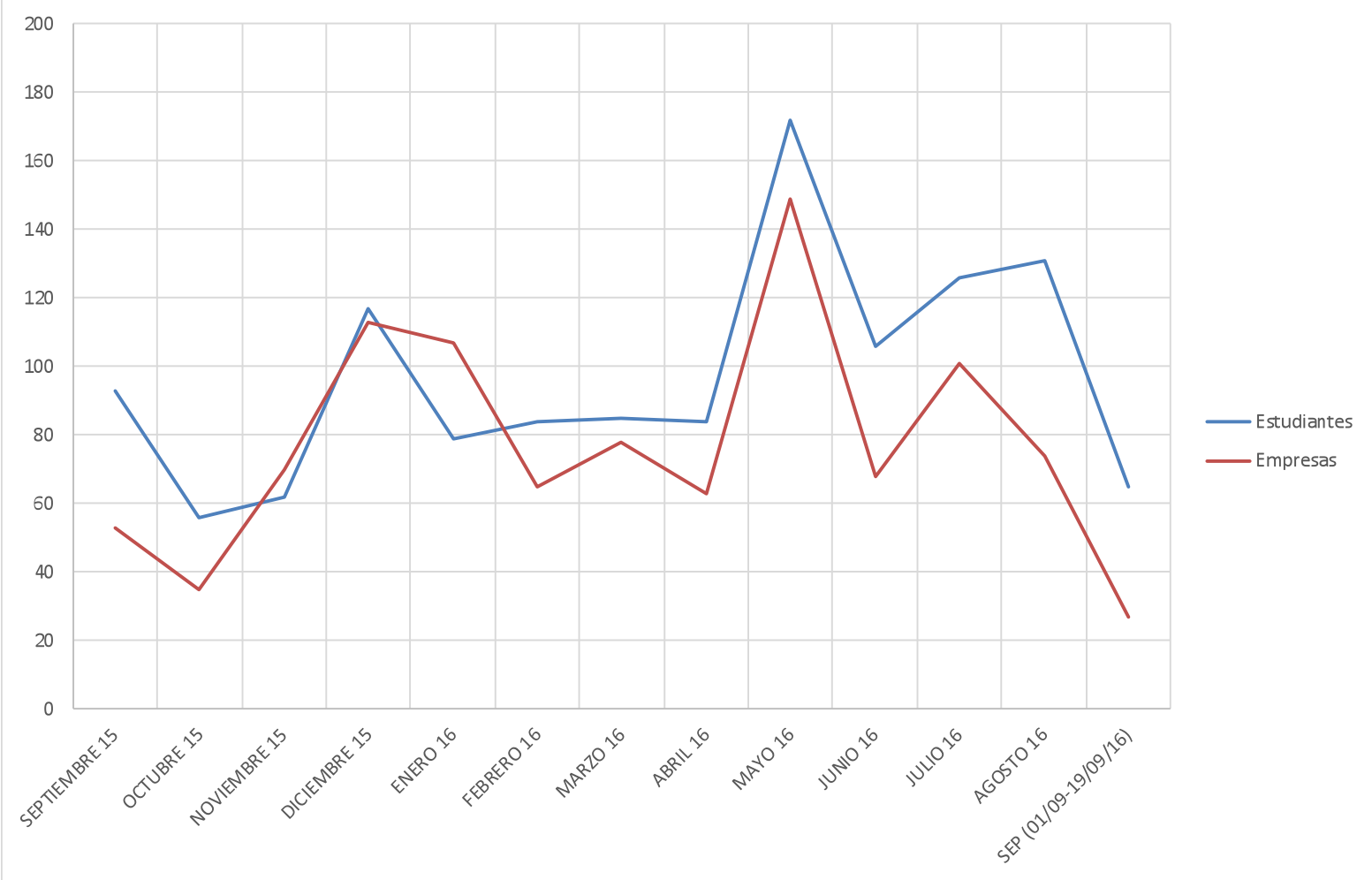

Figura 13. Evolución mensual del número de informes finales. Elaboración propia.

\section{Análisis y resultados}

Las encuestas se plantearon como parte de los informes finales tanto del estudiante como de la empresa de forma que resultase más fácil completarla. Se trata de encuestas cortas ( 7 preguntas para estudiantes y 3 para empresas), con cuestiones directas sobre la adecuación de los contenidos, los conocimientos y la satisfacción general por ambas partes. Debemos señalar que el módulo de encuestas de la plataforma ICARO no permite realizar distinciones 
entre contenidos diseñados de forma separada para prácticas curriculares o extracurriculares, por lo que la encuesta utilizada es la misma que la utilizada para las prácticas curriculares que se gestionan a través de la plataforma. A continuación, se muestran los resultados obtenidos tanto en estudiantes como empresas para el período estudiado.

\subsection{Resultados en estudiantes}

La figura 14, muestra el tipo de encuesta utilizada. Se ha optado por una encuesta corta y directa con valoración cerrada según escala Likert. El cálculo de resultados se ha efectuado mediante la extracción del promedio de las valoraciones realizadas por el estudiante.

1. Mi formación era la adecuada para las tareas encomendadas.

2. El/la tutor/a asignado por la empresa supervisaba mi trabajo.

3. El/la tutor/a asignado por la Universidad ha estado disponible cuando lo he necesitado y ha respondido todas las dudas surgidas durante la realización de mis prácticas.

4. Las líneas de trabajo asignadas en $\mathrm{mi}$ proyecto formativo se han cumplido.

5. Las actividades realizadas tienen relación con mi formación.

6. Estoy mejor preparado/a para insertarme en el mundo laboral después de mis prácticas.

7. La formación recibida en mi titulación se adecúa a la que las empresas demandan.

Esta pregunta se valora mediante escala Likert con un rango 1-10, siendo 1 el menor grado de satisfacción y 10 el máximo.

\begin{tabular}{|l|l|l|l|l|l|l|l|l|l|}
\hline 1 & 2 & 3 & 4 & 5 & 6 & 7 & 8 & 9 & 10 \\
\hline \multicolumn{2}{|l|}{} \\
\hline
\end{tabular}

Figura 14. Modelo de encuesta para estudiantes. Elaboración propia.

Analizando por separado los resultados obtenidos para cada pregunta, podemos establecer una alta satisfacción general del estudiante con sus prácticas, con una valoración superior a 7 en todas las cuestiones planteadas.

Así los estudiantes consideran muy adecuada la formación que reciben para las tareas que les planteaban en la empresa $(8,27)$ y se muestran muy satisfechos tanto con la atención que le dedican sus tutores en la empresa $(8,56)$ como con el cumplimiento del proyecto formativo y las tareas que inicialmente se le asignan $(8,40)$. 


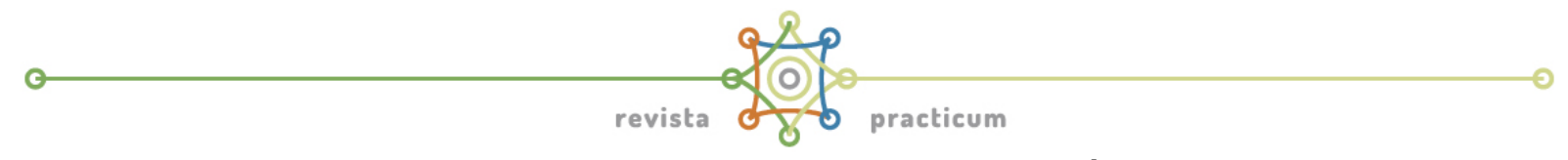

La pregunta peor valorada $(7,07)$ tiene relación con la labor del tutor asignado por la Universidad. Debemos destacar aquí que las tutoras apreciaron que los estudiantes valoraban el servicio negativamente, en algunos casos, alegando que no lo habían utilizado o que lo desconocían.

Igualmente, si bien la pregunta evalúa sólo el trabajo de tutorización, el estudiante responde sobre la percepción general que tiene de los servicios que le presta la Universidad en la gestión administrativa de su práctica. Debemos destacar que la pregunta mejor valorada es la que hace referencia a la cuestión 6 , en concreto la que se refiere a que después de realizar sus prácticas se encuentran mejor preparados para insertarse en el mundo laboral $(8,67)$.

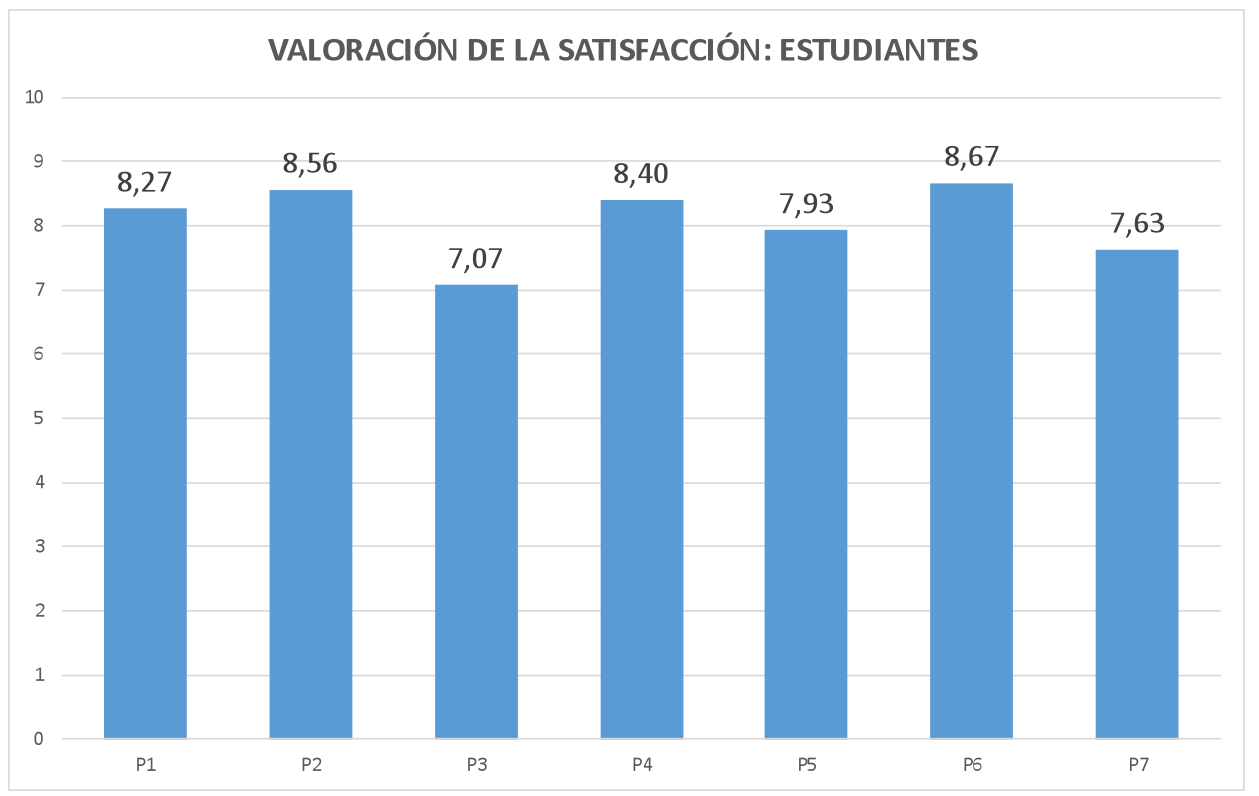

Figura 15. Satisfacción por pregunta (estudiantes). Elaboración propia.

\subsection{Resultados en empresas}

El cuadro muestra el tipo de encuesta utilizada. Se ha optado por una encuesta corta y directa con valoración cerrada según escala Likert. El cálculo de resultados se ha efectuado mediante la extracción del promedio de las valoraciones realizadas por los tutores designados por las empresas. 
1. Indique el grado de satisfacción general con esta práctica.

2. Indique su satisfacción con el nivel de conocimientos técnicos mostrado por el estudiante.

3. Valore la comunicación mantenida con el/la tutor/a en la Universidad.

Esta pregunta se valora mediante escala Likert con un rango 1-10, siendo 1 el menor grado de satisfacción y 10 el máximo.

\begin{tabular}{|l|l|l|l|l|l|l|l|l|l|}
\hline 1 & 2 & 3 & 4 & 5 & 6 & 7 & 8 & 9 & 10 \\
\hline \multicolumn{1}{|l}{} \\
\hline
\end{tabular}

Figura 16. Modelo de encuesta para empresas. Elaboración propia.

Analizando por separado los resultados obtenidos para cada pregunta, podemos establecer una alta satisfacción general de la empresa con los estudiantes que realizan sus prácticas, con una valoración superior a 7 en todas las cuestiones planteadas.

Así llama la atención la valoración de la satisfacción con la práctica $(8,93)$ y se muestran muy satisfechos con el nivel de conocimientos mostrado por los estudiantes $(8,58)$. Sin embargo, al igual que ocurre con los estudiantes, esta valoración baja ligeramente cuando se les pregunta sobre la comunicación que han mantenido con el tutor asignado por la Universidad $(7,11)$.

Así, si bien la pregunta evalúa sólo el trabajo de tutorización, la empresa responde sobre la percepción general que tiene de los servicios que le presta la Universidad en la gestión administrativa de las prácticas. 


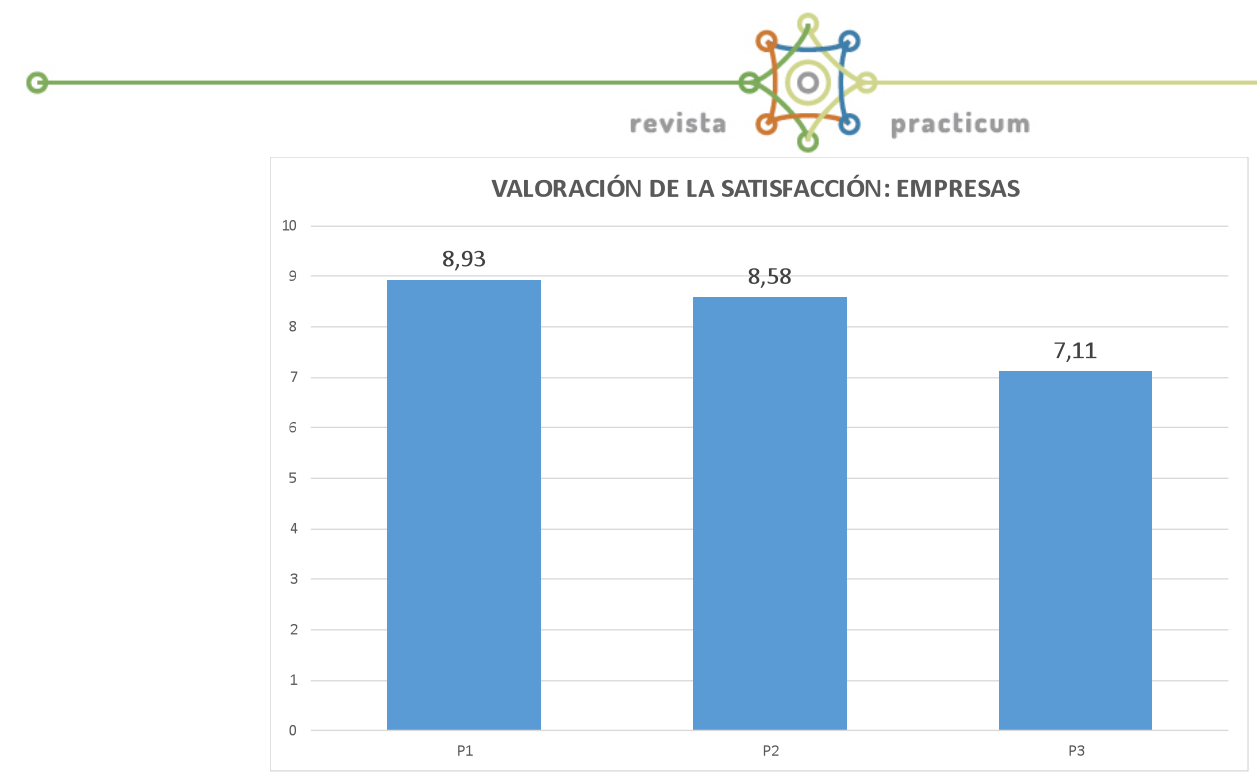

Figura 17. Satisfacción por pregunta (empresas). Elaboración propia.

Para concluir, se ha calculado el promedio general de la satisfacción, tanto de empresas como de estudiantes, con las prácticas externas extracurriculares y se han obtenido unos resultados muy satisfactorios valorando ambas experiencias por encima de 8 puntos.

Para las empresas la satisfacción las prácticas es de 8,21 mientras que en los estudiantes la valoración es ligeramente inferior, puntuando con 8,07 .

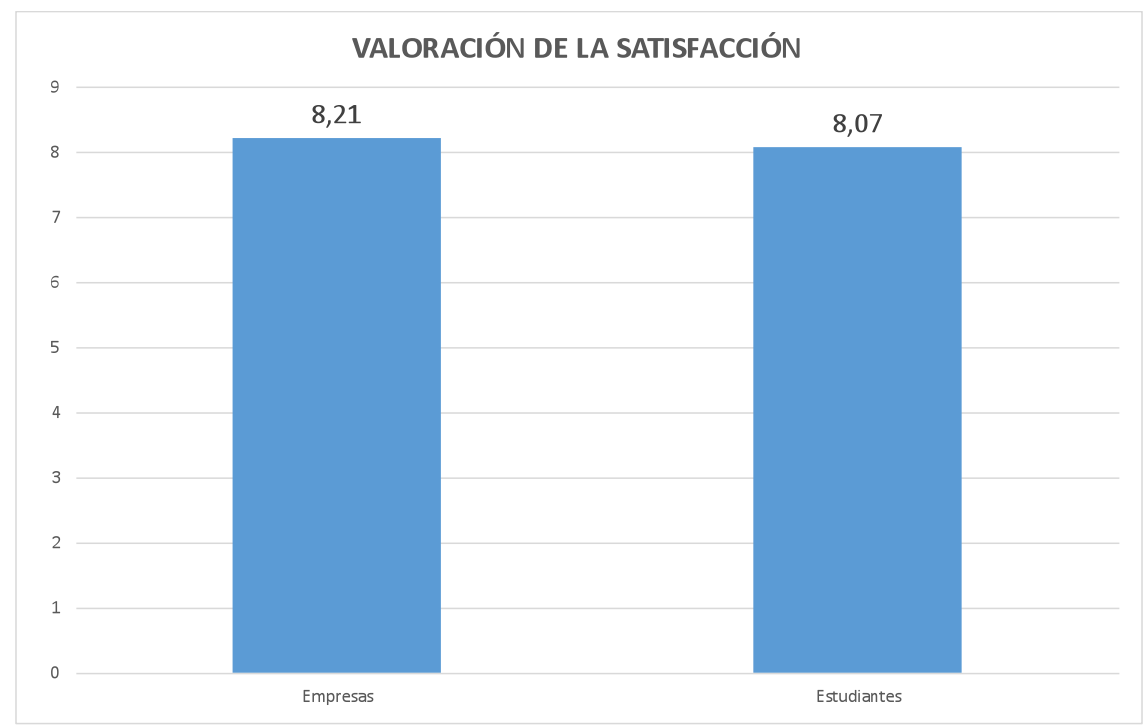

Figura 18. Resultados generales de satisfacción. Elaboración propia. 


\subsection{Cuestiones y dudas frecuentes encontradas en el proceso de tutorización}

Las atenciones directas permitieron que las tutoras recogieran, a modo de diario, las cuestiones y preguntas más frecuentes que atendían. Así las consultas planteadas se referían principalmente a:

- Cotización en la Seguridad Social, derecho a desempleo, compatibilidad con la inclusión en pólizas familiares privadas, pérdida de pensiones o ayudas, falta de diferenciación entre tarjeta sanitaria y número de seguridad social, forma de obtención del número de la Seguridad Social.

- Bolsa de estudio, obligatoriedad y forma de abono de la ayuda, importe de la misma, retenciones aplicadas.

- Derechos laborales, permisos, vacaciones, pagas extras... suelen confundir sus derechos con los de los trabajadores de la empresa.

- Duración de la jornada de prácticas, reparto de la jornada, horas a realizar diariamente, horarios, etc.

- Reconocimiento de las prácticas, número de créditos reconocidos, plazos para la emisión de certificados, cuestiones administrativas relacionadas con la certificación de su práctica.

Las atenciones personales han permitido detectar problemas concretos relacionados con trato inadecuado al estudiante, negativas por parte de la empresa a realizar la certificación al estudiante, problemas con el pago de la dotación económica, bajas por accidente, falta de adecuación entre el proyecto formativo y las tareas efectivamente asignadas al estudiante, discrepancias entre el estudiante y la empresa...

En estos casos la mediación de las tutoras entre estudiante y empresa facilitó la resolución de los casos.

\section{Conclusiones}

La experiencia ha permitido, por un lado, dar cumplimiento al precepto reglamentario que se contiene en el R.D. 592/2014, de 11 de julio, por el que se regulan las prácticas académicas externas de los 
revista

estudiantes universitarios, y por otro dar respuesta a las demandas de datos surgidas de los procesos de acreditación de los títulos universitarios con relación al nivel de satisfacción de las prácticas extracurriculares tanto de estudiantes como de sus potenciales empleadores.

La puesta en marcha del programa ha supuesto un acercamiento tanto a los estudiantes como a las empresas, creando un vínculo mucho más directo e incrementando la calidad del servicio que se ofrece.

Al incrementar el control sobre el desarrollo de las prácticas extracurriculares se producen una mayor equiparación con las curriculares, tradicionalmente más tutorizadas, de forma que se facilita el reconocimiento de unas por otras, mejorando la capacidad de oferta de los Centros.

La tutorización ofrece información relevante para detectar tanto fortalezas como deficiencias en los procedimientos de gestión administrativa de las prácticas.

Así, si bien el resultado general es muy satisfactorio debemos trabajar en las siguientes líneas:

- Mejora de la oferta en titulaciones con escasa oferta de prácticas. Se debe trabajar para disminuir la brecha entre titulaciones, así como fomentar el conocimiento de las competencias asociadas a las titulaciones de Posgrado, menos demandadas por las empresas, en la mayoría de los casos por desconocimiento.

- Mejor difusión de la información. Se aprecia un gran desconocimiento, tanto en empresas como en estudiantes, de la normativa aplicable, condiciones, características de las prácticas, derechos y obligaciones.

- Mejora de la formación del personal asignado a la Unidad de Prácticas en cuestiones que, si bien no son de su competencia directa, sí resultan de muy frecuente consulta. Estas cuestiones son principalmente las que tienen relación con la Seguridad Social.

- Mejora del procedimiento administrativo de gestión de prácticas de forma que se acorten tiempos y se dé una mejor respuesta que permita incrementar el nivel de satisfacción. 
- Modificación de la Normativa de Prácticas Externas de la UMA. Fruto de la experiencia de los últimos años, se hace necesario "pulir" la normativa en aspectos que permitan acotar aspectos que son fuente de queja frecuente (limitación de la jornada, aclaración sobre permisos, vacaciones, bajas, interrupciones, régimen de titulaciones propias, limitación de la duración máxima de las prácticas, así como de sus prórrogas...).

Mejora del sistema de tutorización. El procedimiento actual requiere una gran dedicación y no resulta fácil la extracción de los datos. Se debe seguir trabajando en la reformulación de las cuestiones y en la mejora de la recogida de datos.

\section{Referencias}

Universidad de Málaga, I. (2017). Universidad de Málaga. Uma.es. Retrieved 10 January 2017, from https://goo.gl/aivOzl

Ministerio de la presidencia, I. (2011). Real Decreto 1707/2011, de 18 de noviembre, por el que se regulan las prácticas académicas externas de los estudiantes universitarios. https://goo.gl/b409IO

Ministerio de la presidencia, I. (2014). Real Decreto 592/2014, de 11 de julio, por el que se regulan las prácticas académicas externas de los estudiantes universitarios. https://goo.gl/fMF2Sa

Ministerio de la presidencia, I. (1981), Real Decreto 1497/1981, de 19 de junio, sobre Programas de Cooperación Educativa. https://goo.gl/dZA19Y

Ministerio de la presidencia, I. (2010) Real Decreto 1791/2010, de 30 de diciembre, por el que se aprueba el Estatuto del Estudiante Universitario. https://goo.gl/aGA0G7 